\title{
Advanced SIMION Techniques: Boundary Matching and Genetic Optimization
}

\author{
D. Dowsett
}

Department "Science and Analysis of Materials" (SAM), Centre de Recherche Public - Gabriel Lippmann, Belvaux, Luxembourg.

\begin{abstract}
Two techniques for use with SIMION [1] are presented, boundary matching and genetic optimization. The first allows systems which were previously difficult or impossible to simulate in SIMION to be simulated with great accuracy. The second allows any system to be rapidly and robustly optimized using a parallelized genetic algorithm. Each method will be described along with examples of real world applications.
\end{abstract}

\section{Boundary Matching}

Modelling systems which involve multiple different length scales is difficult in SIMION. As each potential array (PA) has a fixed mesh size it is not possible to optimize the mesh for different regions. A method for overcoming this kind of problem by using multiple overlapping PAs with varying mesh sizes is presented. The potential on boundary of a PA is matched to that of the surrounding PAs such that ions/electrons crossing the boundary see no discontinuities in potential. While this type of approach has been used elsewhere [2,3], it does not seem to be widely used by the SIMION community. This is somewhat surprising as it is an incredibly versatile technique that allows systems and effects that were previously impossible to simulate accurately in SIMION to be modelled. The aim of this paper is to showcase the method and demonstrate its versatility with some practical examples of systems that cannot be modelled without it. The method may be used with both standard PA files and with fast adjustable PA0 files.

The classic example of a system with two drastically different length scales is a field emitter inside an extraction system. The apex radius of the field emitter can be of the order of a few nanometres depending on the type (LMIS, Schottky, cold field), where-as the extraction electrode is typically a few tens of $\mathrm{mm}$ in diameter. This leads to a scale ratio of $\sim 10^{6}$. Most CPO software handles this problem by using a variable mesh density or multiple meshes. To solve the problem in SIMION, software was developed (initially in $\mathrm{C}++$ but now in LUA) to programmatically create a series of nested PAs with increasing mesh density with the potential on the boundary of each array copied and interpolated from the surrounding lower density array. The process described below is shown schematically in Figure 1 for the example of a liquid metal ion source (LMIS) next to a plane extraction electrode.

1. A geometry file of the system is created. This can be done in such a way that only a single geometry file is required to create all the PAs.

2. The lowest resolution PA is created and refined.

3. For the zone of interest, a new higher density PA is created and the potential along the border of the high resolution PA is copied from the corresponding region in the low resolution array.

4. The electrode geometry is added.

5. The high resolution array is refined.

6. The process is repeated until the desired mesh density has been reached.

7. The PAs are arranged on the workbench. 


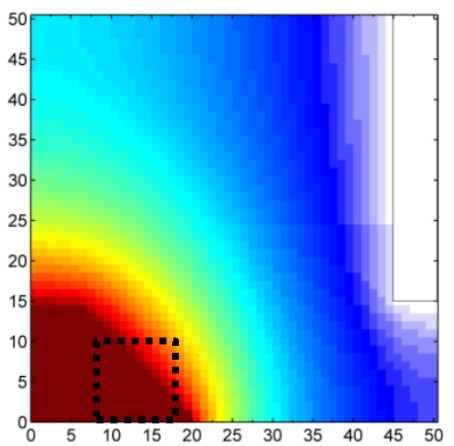

Step 2

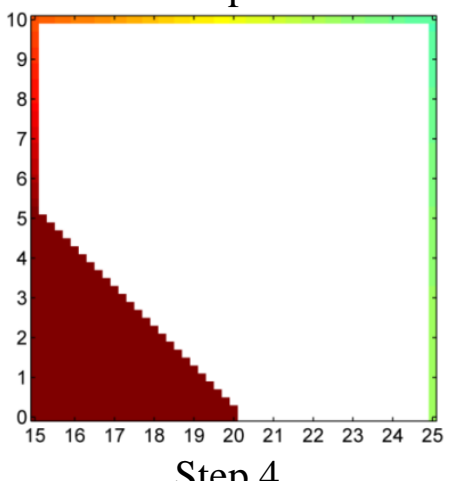

Step 4
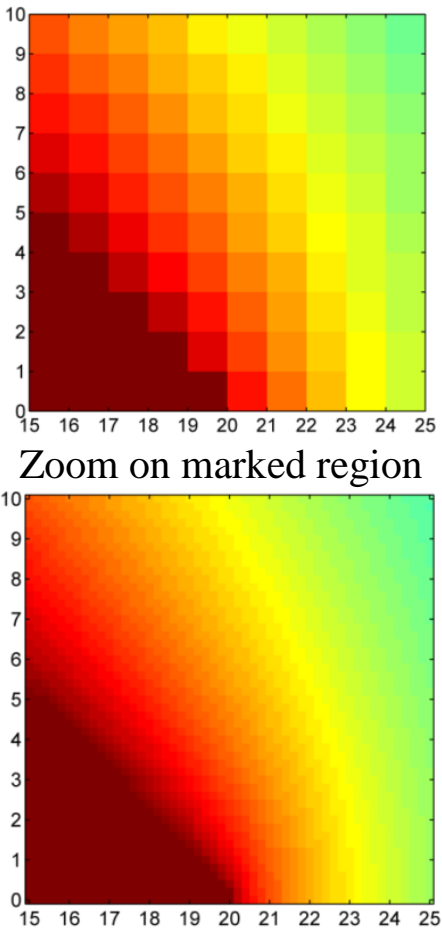

Step 5

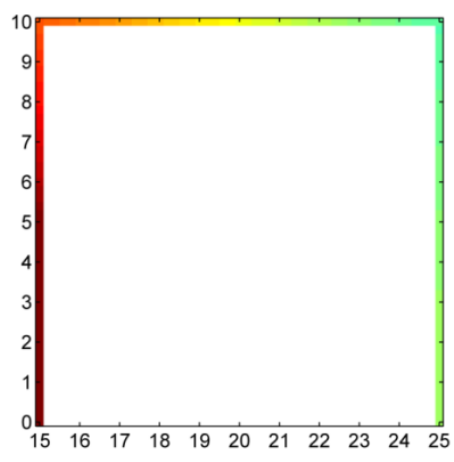

Step3

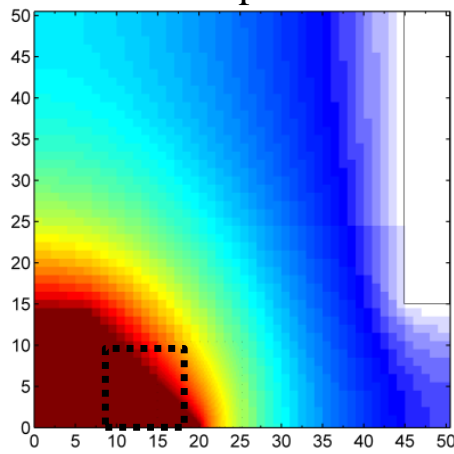

Step 7

Figure 1. Schematic of the boundary matching process for an LMIS next to a planar extraction electrode. Only one matching stage is shown, resulting in a 10x in mesh density over the zone of the LMIS tip.

To illustrate the technique, an LMIS system was modelled. The geometry of the system is shown schematically in Figure 2a. To model the LMIS, the hemisphere on cusp model of Kingham and Swanson [4] was used with dimensions taken from [5]. A series of 7 PAs centred on the apex of the cusp $(\mathrm{Z}=15 \mathrm{~mm}$ ) each with $10 \mathrm{x}$ higher mesh density was created and arranged on the workbench. The highest mesh density was $100 \mathrm{gu} / \mathrm{nm}$. The radius of the cusp was deliberately made a few grid units undersize and ions flown from an equipotential surface at the correct radius. This way the jaggedness of the cubic mesh does not affect the ions initial trajectories. To demonstrate that the potential varies smoothly as ions crosses from on PA to the next, the local potential was stored at every time step of the trajectory integration. Figure $2 \mathrm{~b}$ shows a plot of potential versus position for an ion flown along the axis. No discontinuities are observed at PA boundaries. The inset shows the potential as the ion traverses the region $\pm 1.5 \mathrm{gu}$ around the first boundary transition at $Z=15.0000025$. To more accurately model LMIS emission, space charge effects would have to be taken into account, which is beyond the scope of this paper. However, his example shows that systems with drastically different length scales may be modelled in SIMION.

The boundary matching technique has a wide range of potential applications; ensuring smooth transitions across boundaries in standard simulations; systems with different characteristic length scales; reducing aliasing close to electrodes that are tilted with respect to the mesh; accurately modelling nonideal grids; and reducing the memory required for large scale simulations. A real world example combining the problems of drastically different length scales and regions of different symmetry comes from investigating how the surface topography of a sample can introduce artefacts into Secondary Ion 
Mass Spectrometry (SIMS) images. To perform SIMS analysis a sample is immersed in an extraction field which collects the secondary ions liberated by primary ion bombardment. However, the topography of the sample modifies the local electric field and hence the initial trajectories of the secondary ions [6].

(a)

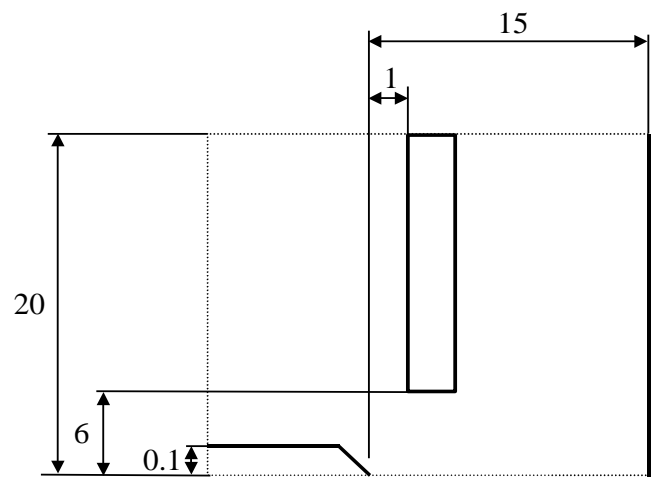

Figure 2. (a) Schematic of LMIS geometry. (b) Potential versus position for an ion flown along the optic axis of the extraction system. (b)

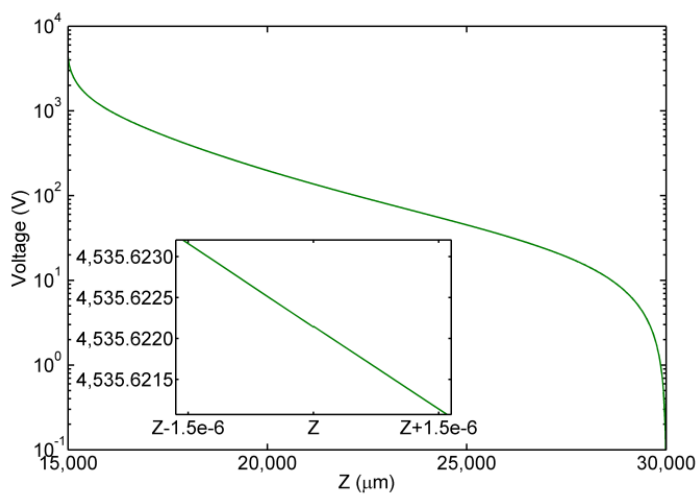

This can significantly modify the collection efficiency across the area of interest. To investigate this and other effects we have modified our Cameca NanoSIMS 50 to include an in-situ AFM [7,8]. This allows the topography of the sample to be measured before, during and after SIMS analysis. The surface topography can then be transformed into a PA in SIMION and added to a model of the extraction system of the NanoSIMS to investigate how the collection efficiency varies across the surface of the sample. The local topography can be on the nanometre scale and has arbitrary geometry, where-as the extraction system has millimetre scale dimensions and is rotationally symmetric. This problem can be solved by creating two boundary matched potential arrays. The first with rotational symmetry and low mesh density, containing the extraction electrodes and the second with planar symmetry and a mesh density appropriate for the sample topography. The top surface of the high density array is boundary matched with the rotationally symmetric array. As the exact position of the sample in the extraction system is known from the SIMS measurement, it is not assumed that the sample is perfectly centred with respect to the rotation axis of the extraction system. Monte Carlo techniques may then be used to launch secondary ions from the surface of the sample with representative angular and energy distributions.

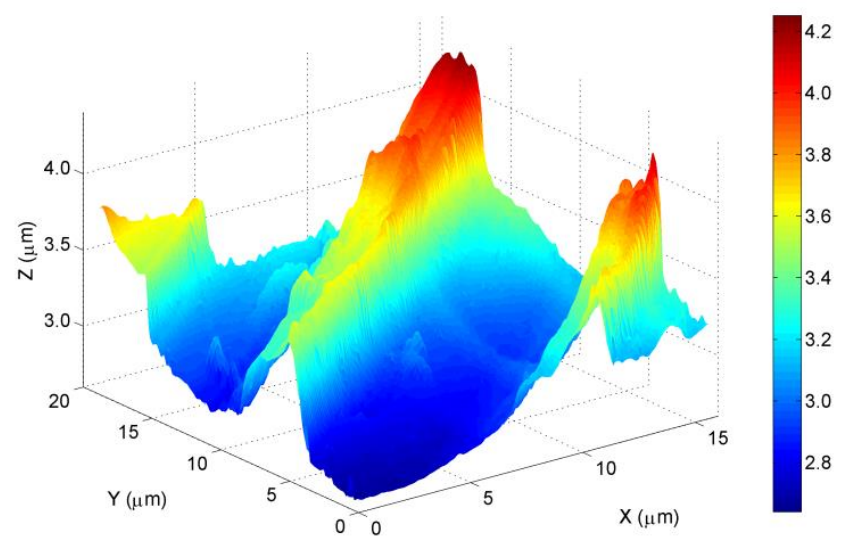

(a)

(b) slice re-coloured according to relative collection efficiency of the NanoSIMS. 
Figure 3a shows a single AFM slice of a test sample (Al layer on $\mathrm{Cu}$ substrate) taken before SIMS analysis. The roughness is of the order of $2 \mu \mathrm{m}$ over the field of view of $15 \times 15 \mu \mathrm{m}^{2}$. Figure $3 \mathrm{~b}$ shows the same sample coloured according to the relative collection efficiency of the extraction system. It can be seen that areas with high aspect ratios lead to a reduction in collection efficiency as the local field curvature is high, deflecting the secondary ions away from the extraction system. The relative collection efficiency drops from 1 in the flat areas on either side of the ridge to 0.4 on its surface to less than 0.1 on its sides. This will manifest itself in SIMS images as edges appearing less bright than they should. This investigation is a first step in attempting to understand and correct topography dependent artefacts in SIMS images. Other effects that need to be taken into account are local variations in work function and angle dependent differential sputtering.

\section{Genetic Algorithm}

Optimization of complex ion/electron optical systems is a difficult and computationally demanding task. While SIMION includes a simplex optimizer that works for simple systems, for complex multiparameter optimizations it is not sufficient. Genetic algorithms (GA) are a well established, robust optimization technique $[9,10]$. They efficiently solve maximization or minimization problems, especially where the shape of the problem space is complex. They have the advantages of being parallelizable, providing good solutions, even when very large problem spaces are used and, under the right circumstances, successfully finding global maxima or minima. Despite their advantages they have so far only rarely been applied to problems in charged particle optics [11,12].

Genetic algorithms work by mimicking the process of evolution. They consist of a population of individuals, where each individual has a strand of DNA which encodes one set of parameters in the problem space. A fitness function is used to evaluate each member of the population. The fitness function evaluates how well a given set of parameters solves the problem. For a detailed description of how genetic algorithms work see Refs. 9 and 10. A parallelized genetic algorithm has been written for use with SIMION. The GA is split into three parts: a gui written in MS Visual C++, a database which stores the population and a series of SIMION workers which evaluate the fitness of each individual. Splitting the GA this way ensures that it is fast, robust and scalable to the platform on which it's being run. Figure 4 shows schematically the program flow of the GA and the communication between the different elements. Each of the major modules is described below.

\section{a. Genetic Algorithm}

The GA presented here is relatively standard. A parameter file containing control parameters (number of parameters of optimization, range of each parameter, mutation and cross breeding probabilities) for the GA is read in along with an optional seed file. The seed file contains a list of individuals known a priori to have high fitness. The GA creates the initial population and writes it to the database. Individuals are then evaluated by SIMION workers. The GA uses tournament selection to determine which individuals survive, these are then cross bred and mutated to the form next generation. Elitism ensures that the best solutions are never lost. The GA continues to evolve the population until either the convergence criterion for fitness is met, or the maximum number of generations is reached.

\section{b. SQL Database}

To ensure the communication across networks is robust, the population is maintained in a separate database. The database keeps track of the fitness of each member of the current generation. Database queries (read/write fitness, compare individuals etc.) are made by the GA and SIMION worker using 
SQL commands. The next generation is appended to the list of individuals, this way the complete evolution of the population may be inspected at any time.

\section{c. SIMION worker}

To allow for parallel evaluation of individuals the SIMION worker structure was developed. Each worker is a copy of SIMION running in batch mode that polls the database to see if there are any individuals to be evaluated. After evaluation of an individual the worker re-polls the database looking for unevaluated individuals. The worker has two parts, a standard lua framework which handles communication with the database and the control program for the ion optics bench which controls all aspects of the simulation including fitness determination. The key advantage of this system is that network communication allows any number of workers to be run on any number of machines. This results in a linear speed up of the optimization with the number of workers.

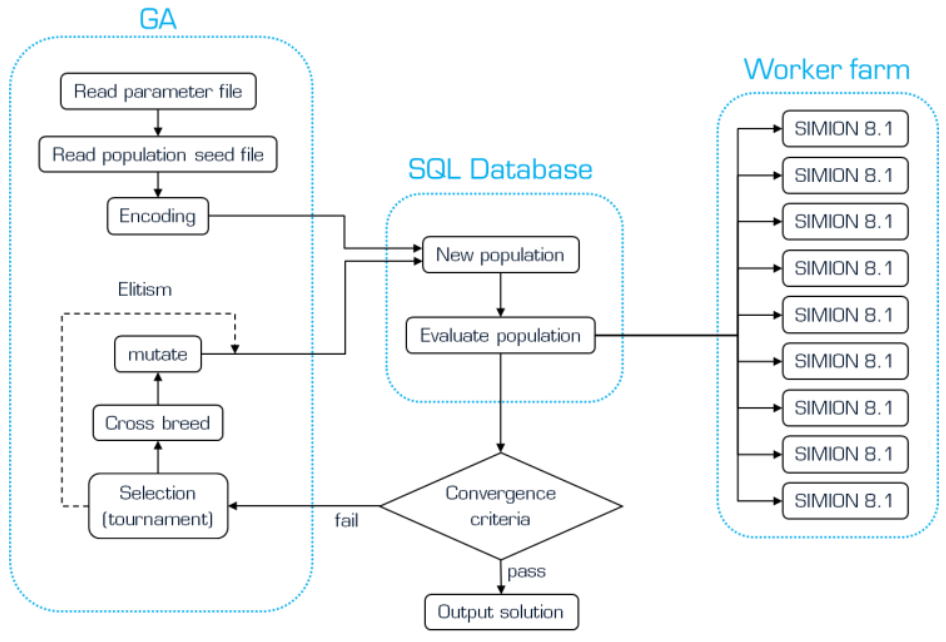

Figure 4. Flow diagram for the genetic algorithm.

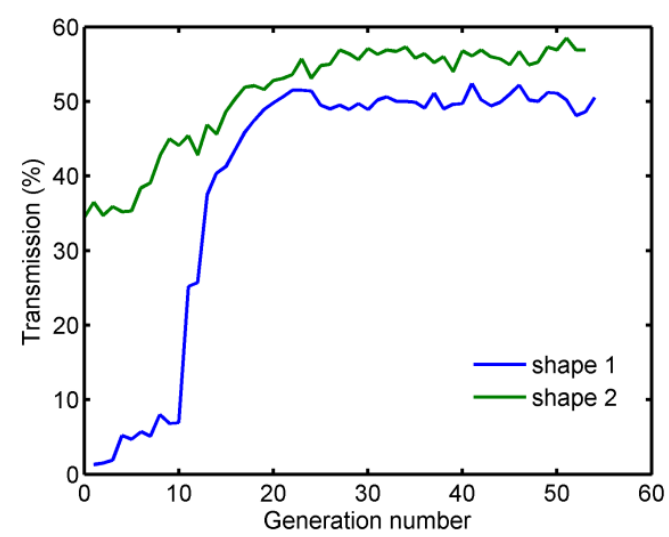

Figure 5. Optimization of TEM high voltage sample holder shape.

Several benchmark tests were performed to ensure the algorithm was working correctly before attempting to tackle a more challenging problem. Our group is in the process of adding a SIMS spectrometer to a TEM to perform SIMS based correlative microscopy [13]. One of the most important aspects of the development was the design of a high voltage sample holder for the TEM. Previous investigations by the author [14] have shown that shaping the extraction field to provide a lensing effect can lead to higher transmission through a spectrometer and therefore increased sensitivity. The aim of the study was to compare sample holders of different shapes and maximize the transmission through the complete mass spectrometer. However, the proximity of the TEM pole pieces to the sample results in an inhomogeneous electric field above it, tilting the secondary ions away from the axis of the extraction system. This tilt requires correction by a quadrupole. After passing through the extraction system, the beam must be focused on the entrance plane of the spectrometer by a two lens transfer optic. However, both the size of the tilt correction and the correct focussing voltages are dependent on the shape of the sample holder, making proper comparison difficult as the system must be correctly optimized for each case. The genetic algorithm was used to optimize the transmission of the spectrometer (at fixed mass resolution) for each sample holder shape (not shown). To further demonstrate the capability of the genetic algorithm, no prior knowledge of the correct focusing voltage for the transfer or spectrometer lenses was used, resulting in a problem with 4 parameters $\left(\mathrm{V}_{\text {Lens } 1}: 2000-4500 \mathrm{~V}, \mathrm{dV}=1 \mathrm{~V}\right.$; $\mathrm{V}_{\text {quad: }}: 0-50 \mathrm{~V}$, $\mathrm{dV}=0.1 \mathrm{~V}$; $\mathrm{V}_{\text {Lens2 }}: 2000-4500 \mathrm{~V}, \mathrm{dV}=1 \mathrm{~V}$ and $\mathrm{V}_{\text {spectrometer }}: 2000-4500 \mathrm{~V}, \mathrm{dV}=1 \mathrm{~V}$ ) and a parameter space of $3 \times 10^{11}$ possible values. To perform the simulation, a complete model of the IMS $4 \mathrm{f}$ including 
the modified extraction system and near sample region of the TEM was built. The transmission was determined by launching a bundle of 1000 ions with initial positions generated uniformly over a $1 \mu \mathrm{m}$ field of view. Monte Carlo techniques were used to generate angular and energy distributions representative of secondary ions sputtered by a $30 \mathrm{keV} \mathrm{Ga}$ primary beam. The transmission was determined by counting the number of ions reaching the detector with the mass selection slit set to provide a mass resolving power of 1500 .

The evolution of the best individual for two different sample geometries is shown in Figure 5. Both geometries show a significant improvement in transmission ( $50 \%)$ compared with the standard instrument $(\sim 8 \%)$ at this mass resolving power. This is the result of a number of factors including, much smaller than normal analysed area and different magnification of the transfer optics. The voltages found by the GA for $V_{\text {Lens2 }}$ and $V_{\text {spectrometer }}$ are within a few percent of values previously determined experimentally for $4 \mathrm{f}$ spectrometers. The most impressive aspect of the optimization comes when investigating the range of voltage values which lead to any ions hitting the detector. The most critical parameter is $V_{\text {Lens } 1}$ and that this voltage must be within $\sim 2 \%$ of the optimal value before any ions reach the detector. This means that for most of the phase space of the optimization the transmission is zero, but the genetic algorithm is still able to efficiently find a region of high transmission. Very few other optimization techniques and none based on gradients would be able to solve the same problem.

\section{Conclusions}

Two methods for extending the scope of what is possible using SIMION have been presented along with their advantages and practical applications for each. Boundary matching significantly extends the range of problems that can be investigated using SIMION and can be used to improve the accuracy of more standard simulations. Optimization by a genetic algorithm has been demonstrated for a complete instrument and found to work remarkably well given the size and shape of the problem space.

References:

1 D Manura and D Dahl. SIMION 8.0 User Manual (Scientific Instrument Services, Inc., Ringoes, NJ).

2 T Radlička and B Lencová, Ultramicroscopy 108 (2008), p. 445.

3 D Edwards, Nucl. Instrum. Meth. A 645 (2011), p. 285.

4 DR Kingham and LW Swanson, Appl. Phys. A 34 (1984), p. 123

5 RG Forbes, Ultramicroscopy 108 (2008), p. 455.

6 JLS Lee et al, Appl. Surf. Sci. 255 (2008), p. 1560.

7 Y Fleming, Appl. Surf. Sci. 258 (2011), p. 1322.

8 T Wirtz et al, Rev. Sci. Instrum. 83 (2012), 063702.

$9 \mathrm{JH}$ Holland in "Adaptation in Natural and Artificial Systems", (University of Michigan Press, Ann Arbor, 1975) p. 183.

10 DE Goldberg in "Genetic Algorithms in Search, Optimization and Machine Learning", (Addison Wesley, Reading, 1989) p. 412.

11 A Benez, E Plies and W Ziegler, Proceedings of EUREM-11, Dublin, Vol. 1 (1996), p. I-117.

$12 \mathrm{C}$ Gu et al, Proc. of SPIE 4510 (2001), doi: 10.1117/12.451273.

13 T Wirtz, Microsc. Microanal. 19 (S2) (2013), p. 356.

14 D Dowsett, P Philipp and T Wirtz, Nucl. Instrum. Meth. A 645 (2011), p. 120. 